\title{
Hemoptysis as a Presenting Symptom for Metastatic Pancreatic Cancer
}

\author{
Toshimasa Okabe, MD, Leigh Van Vranken, MSIII, and Darren N. Seril, MD
}

\section{Introduction}

Approximately 32,000 pancreatic neoplasms are diagnosed in the United States annually. ${ }^{1}$ Currently, carcinoma of the pancreas is the fourth most common cause of cancer death in both men and women in this country, after cancers of the lung, breast/ prostate, and colon. The most common presenting symptoms of pancreatic cancers are epigastric pain, obstructive jaundice, and weight loss. ${ }^{1}$ However, since pancreatic cancer is frequently metastatic when diagnosed, it may uncommonly present with findings characteristic of the site of spread.

\section{Case Presentation}

A 42 year-old Caucasian female with no significant past medical history presented with a four-month history of daily hemoptysis associated with a productive cough and a two-week history of jaundice and gray stools. The initial evaluation for hemoptysis, performed at an outside hospital approximately three months prior to presentation included a normal chest radiograph and negative PPD. Two weeks prior to admission, the patient presented to an outside hospital complaining of epigastric pain for one week, as well as new onset jaundice and persistent hemoptysis. Per the patient, an abdominal ultrasound performed at that time revealed a pancreatic mass. She presented shortly thereafter to our hospital with worsening hemoptysis, epigastric and right upper quadrant pain, and jaundice. She described the abdominal pain as constant and dull, with an intermittent stabbing component. The pain was aggravated when lying down and relieved by oxycodone-acetaminophen. She also reported generalized fatigue and a five-pound weight loss over three weeks.

She denied fever, chills, night sweats, chest pain, shortness-ofbreath, nausea, vomiting, or diarrhea. She has no known drug allergies. Her only medication was oxycodone-acetaminophen which was started for abdominal pain during the previous hospital visit. She has no history of abdominal or other surgeries. She consumes three to four drinks of alcohol per week during the past two years and has smoked approximately one pack of cigarettes per day for the past 25 years. She used cocaine on occasion more than 10 years ago. She denied intravenous drug use. Her mother, a smoker, died from small cell lung cancer, and her father died from prostate cancer. She denied recent travel, as well as known exposure to individuals with tuberculosis, asbestos, or other inhaled chemicals.

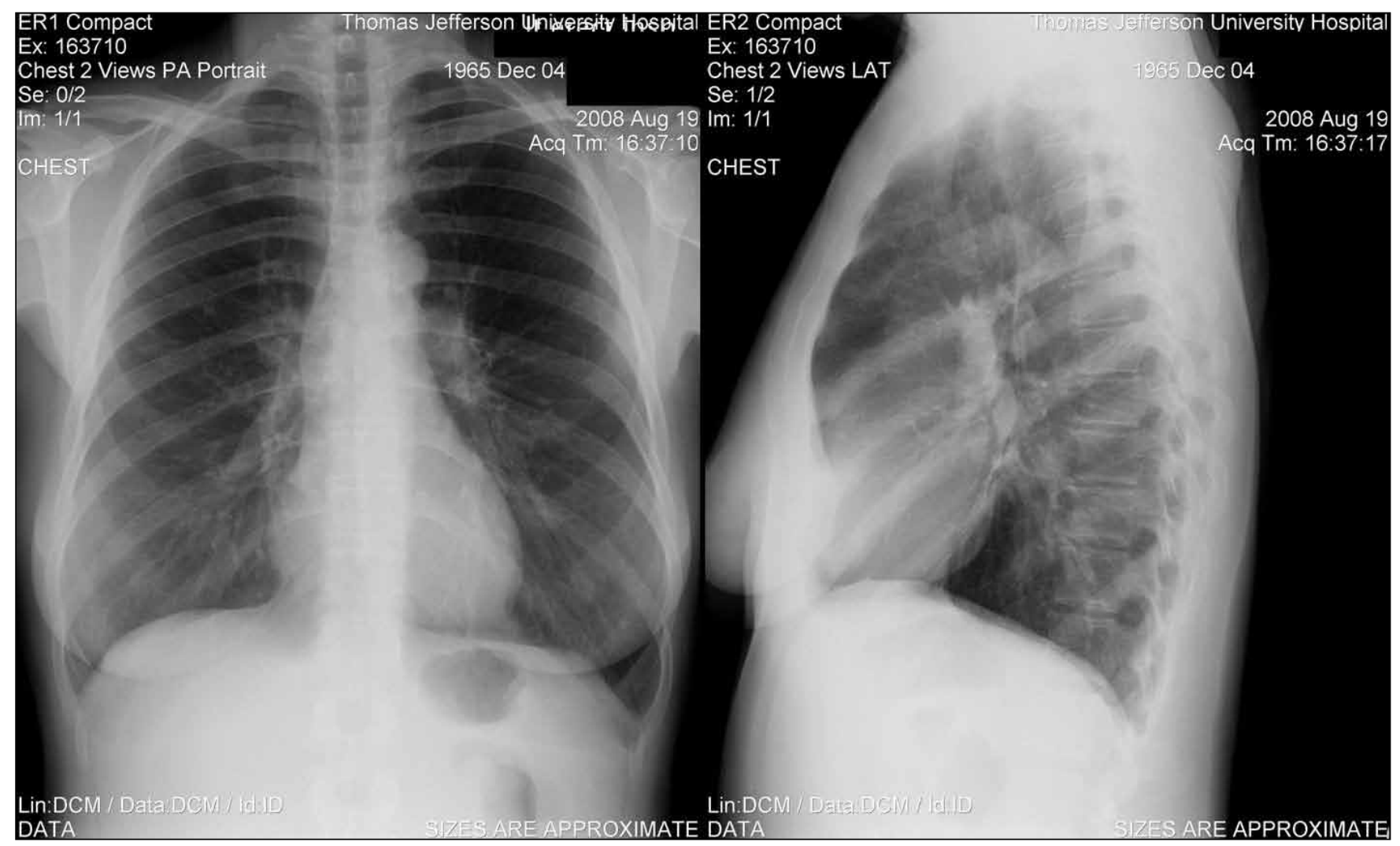

Figure 1. PA and lateral chest radiographs shows showed no evidence of infiltrates, effusions, or masses. 


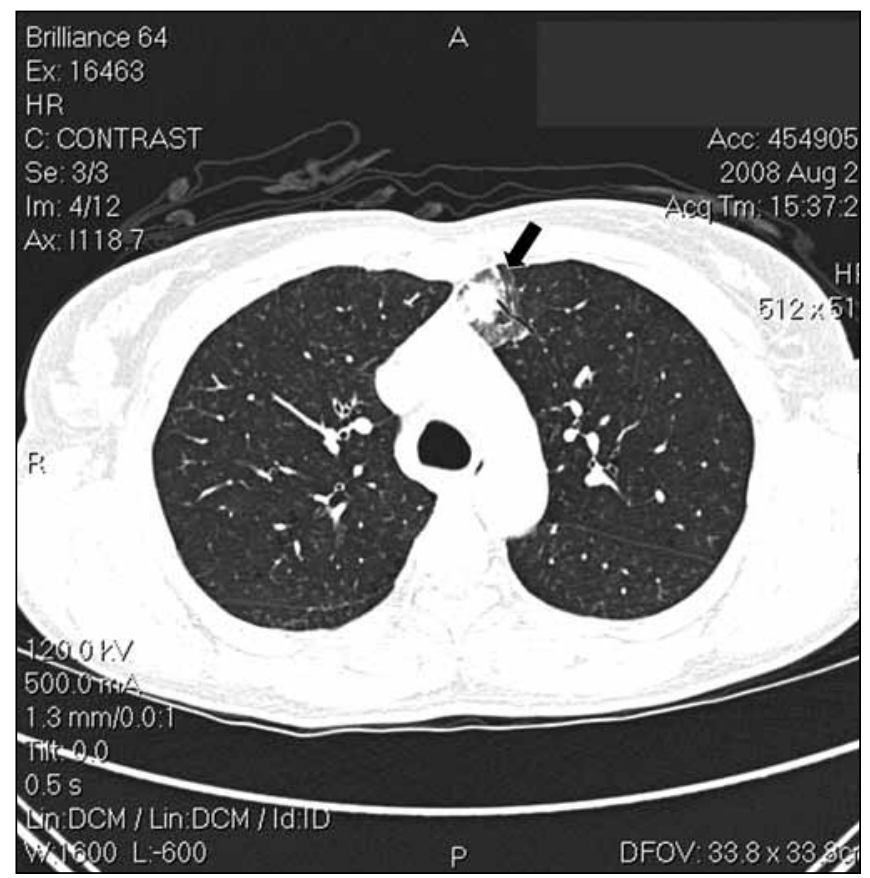

Figure 2. CT thorax with contrast illustrates a cavitary lesion in the anterior segment of the left upper lobe (arrow).

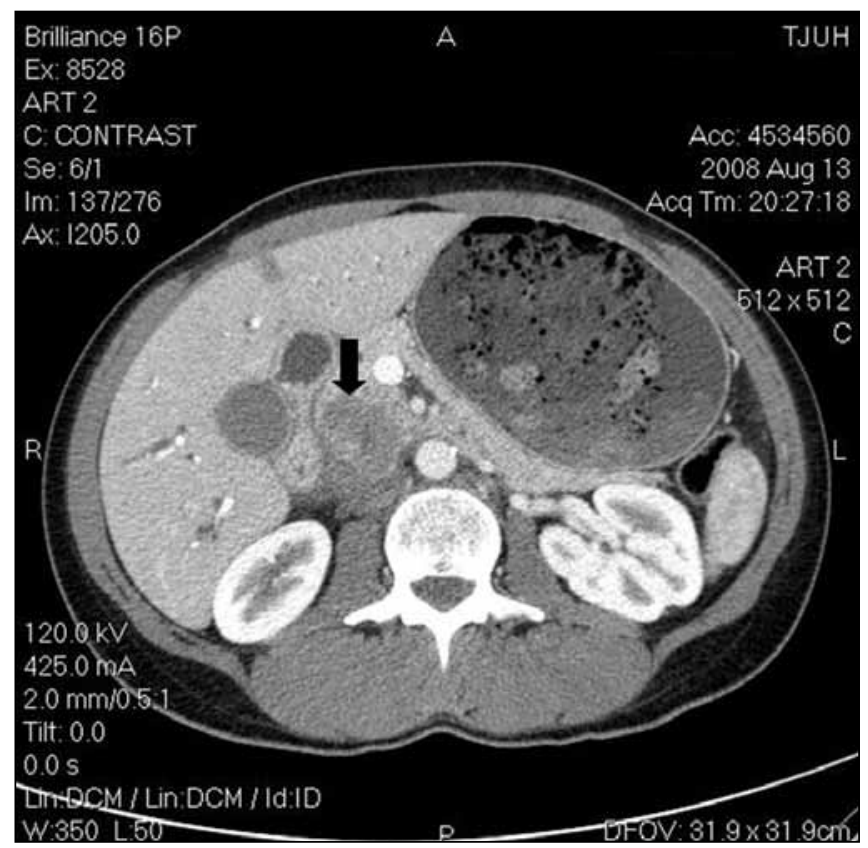

Figure 3. Multi-detector CT abdomen and pelvis with contrast detects a hypodense mass in the head of the pancreas (arrow).

\section{Hospital Course}

Initial examination revealed a jaundiced but well-appearing middle-aged woman in no acute distress. Vital signs revealed a temperature of $98.7^{\circ}$ Fahrenheit, blood pressure $92 / 62 \mathrm{~mm} \mathrm{Hg}$, heart rate 70 beats/minute, respiratory rate of 18 breaths/minute, and oxygen saturation of $96 \%$ while the patient was breathing ambient air. The sclerae were icteric. The lungs were clear to auscultation bilaterally without wheezes, rales, or rhonchi. Abdominal exam revealed tenderness on palpation of the right upper quadrant and epigastrium without rebound tenderness or guarding. No Murphy's sign was detected. The skin was jaundiced without rashes, spider angiomata, or palmar erythema. The remainder of the physical examination was unremarkable.

The results of the laboratory tests were significant for total bilirubin $10.8 \mathrm{mg} / \mathrm{dL}$, direct bilirubin $4.9 \mathrm{mg} / \mathrm{dL}$, AST $148 \mathrm{IU} / \mathrm{L}$, ALT 196 IU/L, and alkaline phosphatase 225 IU/L. CEA was 1.1 $\mathrm{ng} / \mathrm{mL}$ (normal range: $0.0-5.0 \mathrm{ng} / \mathrm{mL}$ ) and CA $19-9$ was $12 \mathrm{ng} /$ $\mathrm{mL}$ (normal range: 0.0-36.0 U/mL). Her complete blood count, basic metabolic panel including creatinine, protein, albumin, amylase, lipase, PT, and PTT were within normal limits.

A chest radiograph obtained on admission showed no evidence of infiltrates, effusions, or masses (Figure 1). A chest CT with contrast performed at an outside hospital two weeks prior to presentation revealed a cavitary lesion in the anterior segment of the left upper lobe. The lesion was confirmed by repeat CT scan (Figure 2) and measured 2.6 X $2.5 \mathrm{~cm}$, with no marked changes in size or appearance from the prior study. Several small lung nodules were noted as well. In addition, enlarged mediastinal lymph nodes measuring $10 \mathrm{~mm}$ in diameter were noted in the hila bilaterally. Multi-detector abdominal CT with contrast (Figure 3) revealed a pancreatic head mass measuring $3.5 \times 3.3 \mathrm{~cm}$, with abdominal lymphadenopathy, and intra- and extra-hepatic biliary ductal dilatation.

Based on these findings, the initial differential diagnosis included a metastatic neoplasm of either pulmonary or pancreatic origin. Disseminated tuberculosis or other bacterial or fungal infection, as well as a vasculitic process, were also considered. Antineutrophil Cytoplasmic Antibody (ANCA) serologies were obtained and were negative. ESR was $22 \mathrm{~mm} / \mathrm{hr}$ (normal range: 0-15 mm/hr) and CRP was $0.7 \mathrm{mg} / \mathrm{l}$ (normal range: $<12 \mathrm{mg} / \mathrm{L}$ ).

For diagnostic and therapeutic purposes, endoscopic retrograde cholangio-pancreatography (ERCP) was performed. A common bile duct (CBD) stricture was noted, and brush cytology of the CBD was obtained. Subsequently, an expandable metal stent was placed in the CBD after sphincterotomy. In order to further evaluate the pancreatic mass, endoscopic ultrasound (EUS) was performed, and fine needle aspiration (FNA) biopsy of the mass was performed. Bronchoscopy was performed to further evaluate the cavitary lung lesion noted on CT scan. Bronchoalveolar lavage (BAL) and bronchial brushings were sent for cytology, Gram stain, and culture. Endobronchial ultrasonography- 


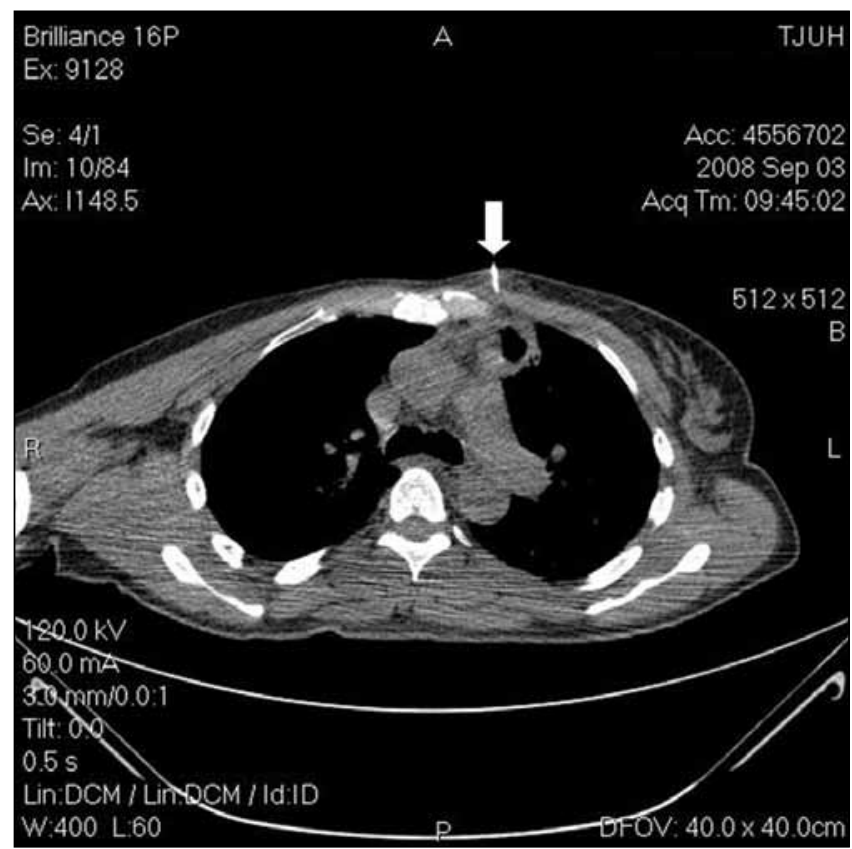

Figure 4. CT-guided biopsy of anterior lung mass.

guided biopsy was attempted; however, the biopsy was unable to be obtained due to excessive coughing during the procedure.

Cytologic analysis of the CBD brushings was negative for malignant cells. However, the FNA of the pancreatic head mass revealed features consistent with pancreatic ductal adenocarcinoma. The microbiology and pathology from the bronchoscopy samples were unrevealing: BAL fluid cultures were negative for bacteria and fungus, and the bronchial brush cytology revealed benign epithelial cells. Thus, a definitive diagnosis of the cavitary lung lesion was lacking.

The patient's jaundice and abdominal pain improved following the placement of the CBD stent. She was discharged home with a presumptive diagnosis of metastatic pancreatic cancer, and arrangements to follow-up with medical oncology. Four days later, the patient was re-admitted for complaints of fever and headache. Brain MRI and bone scan showed no evidence of metastasis to those sites. The patient's hospital course was complicated by E. coli bacteremia, likely secondary due to ERCP and CBD stent placement. Having been treated with intravenous antibiotics, and with subsequent negative blood cultures, she was discharged to home with plans to undergo CT-guided biopsy of the lung lesion as an outpatient. Ten days after discharge, biopsy of the lung mass was successfully performed with CT guidance (Figure 4). Pathology revealed malignant cells morphologically similar to those obtained by FNA of the pancreatic mass. In order to fully elucidate the extent of metastasis, positron emission tomographic scanning was performed, which confirmed the presence of a hypermetabolic mass in the pancreas, as well as lesions within the left upper lobe of the lung, left adrenal gland, and L1 vertebral body. The patient later initiated chemotherapy as an outpatient for palliation of widely metastatic pancreatic cancer. She was given a final diagnosis of primary pancreatic adenocarcinoma metastatic to the lung, adrenal gland, and spine.

\section{Discussion}

Pancreatic cancer is notoriously aggressive. Indeed, surgically resectable cases on presentation represent the minority: approximately $10 \%$ to $15 \%$. Pancreatic neoplasms metastasize by hematogenous and lymphatic routes. Locally, the celiac plexus, superior mesenteric artery, portal vein, and ligament of Treitz are common sites of spread. The liver appears to be the most frequent target of distant metastasis, as well as the peritoneum. The lung is also a common target. Lung metastases were present in greater than $50 \%$ of patients with metastatic pancreatic cancer in one study. ${ }^{2}$

It is rare for metastatic pancreatic cancer to present initially with pulmonary symptoms. Nevertheless, there are case reports of pancreatic cancer "masquerading" as bronchogenic carcinoma, with initial symptoms including cough and dyspnea, as well as those related to paraneoplastic syndromes, i.e., Horner's syndrome. ${ }^{3}$ It has been shown that pancreatic metastases can be radiographically indistinguishable from primary bronchogenic carcinomas, ${ }^{4}$ highlighting the importance of obtaining biopsy for tissue diagnosis and to guide treatment.

The treatment of pancreatic cancer is broadly based on staging of the disease to determine resectability. With the exception of lymph node spread restricted to the surgical field, metastasis renders pancreatic cancer unresectable. The median survival after diagnosis is extremely poor in the case of unresectable pancreatic cancer: approximately three to five months. ${ }^{1}$ Surgical resection via partial pancreaticoduodenectomy (i.e., the Whipple procedure), typically with adjuvant chemotherapy, is the only treatment modality with any meaningful survival benefit. Median survival can be extended to 12 to 18 months with surgery and still further in conjunction with chemotherapy. ${ }^{5}$ The treatment of unresectable pancreatic cancer, including distantly metastatic disease, is essentially palliative and entails management of symptoms, including pain, jaundice, and malabsorption. ${ }^{1}$

The most common findings in the setting of pancreatic cancer include jaundice and abdominal pain. Other findings include Courvoisier's sign (i.e., a palpable nontender gallbladder) and Trousseau's syndrome (i.e., superficial and deep venous thrombosis), as well as more vague symptoms such as fatigue and weight loss. ${ }^{6}$ The presentation is often suggestive of the site of the pancreatic mass. Tumors of the head of the pancreas tend to present with jaundice. Pain is often a later finding, associated with tumors of the pancreatic body and tail. Back pain is an ominous sign, as it may indicate local spread to the 
celiac neuroplexus posterior to the pancreas. The present case is unusual in that pulmonary symptomatology preceded the more typical findings of abdominal pain and jaundice. Because pancreatic head cancers often impinge on the CBD and cause jaundice, these cancers can sometimes be detected at an earlier, less advanced stage than cancers of the body or tail, which are all frequently metastatic to distant sites before pain in the abdomen or the back emerges.

In addition to metastatic pancreatic or lung cancer, our differential diagnosis for the concurrent findings of a cavitary lung lesion and pancreatic mass included atypical infections, such as mycobacterial and fungal infections, other granulomatous diseases, and systemic vasculitides, such as Wegener's granulomatosis. Tuberculosis was a consideration in the present case, although less likely in the context a negative PPD and lack of a suggestive exposure history. Sarcoidosis is well known to involve multiple organs, including the respiratory system, skin, eye, heart, and liver. There are case reports of rare involvement of the pancreas, causing symptoms similar to pancreatic cancer. ${ }^{7}$ Wegener's granulomatosis has been reported to manifest like pancreatic carcinoma as well. ${ }^{8}$ Interestingly, the patient in the present case reported intermittent sinus symptoms, but negative ANCA serologies and a lack of renal involvement made this hypothesis less likely. Ultimately, tissue sampling of the lung and pancreas masses by CT guidance and EUS-FNA proved critical in establishing a definitive diagnosis in this case.
In summary, the current case representsan uncommon presentation of metastatic pancreatic adenocarcinoma, characterized by jaundice and abdominal pain preceded by a prolonged period of respiratory symptoms alone.

\section{References}

1. Ghaneh, P., Costello, E., and Neoptolemos, J.P. Biology and Management of Pancreatic Cancer. Gut 56: 1134-1152, 2007.

2. Kamisawa, T., Isawa, T., Koike, M., Tsuruta, K., and Okamoto, A. Hematogenous metastases of pancreatic ductal carcinoma. Pancreas 11: 345-349, 1995.

3. Cassiere, S.G., McLain, D.A., Emory, W.B., and Hatch, H.B. Metastatic carcinoma of the pancreas simulating primary bronchogenic carcinoma. Cancer 46: 2319-2321, 1980.

4. Steinke, K., Suess, K., and Wiesner, W. Pulmonary metastases from pancreatic adenocarcinoma mimicking bronchoalveolar carcinoma. Eur. Radiol. 10: 1683$1684,2000$.

5. Neoptolemos, J.P., Stocken, D.D., Friess, H., Bassi, C., Dunn, J.A., Hickey, H., Beger, H., Fernandez-Cruz, L., Dervenis, C., Lacaine, F., Falconi, M., Pederzoli, P., Pap, A., Spooner, D., Kerr, D.J., and Büchler, M.W., for the European Study Group for Pancreatic Cancer. A randomized trial of chemoradiotherapy and chemotherapy after resection of pancreatic cancer. N. Engl. J. Med. 350: 12001210, 2004.

6. Barkin, J.S., and Goldstein, J.A. Diagnostic approach to pancreatic cancer. Gastroenterol. Clin. North Am. 28: 709-722, 1999.

7. Harder, H., Buchler, M.W., Frohlich, B., Strobel, P., Bergmann, F., Neff, W., and Singer, M.V. Extrapulmonary sarcoidosis of liver and pancreas: a case report and review of literature. World J.Gastroenterol. 13:2504-2509, 2007.

8. O'Neil, K.M., Jones, D.M., and Lawson, J.M. Wegener's granulomatosis masquerading as pancreatic carcinoma. Dig. Dis. Sci. 37(5):702-4, 1992.

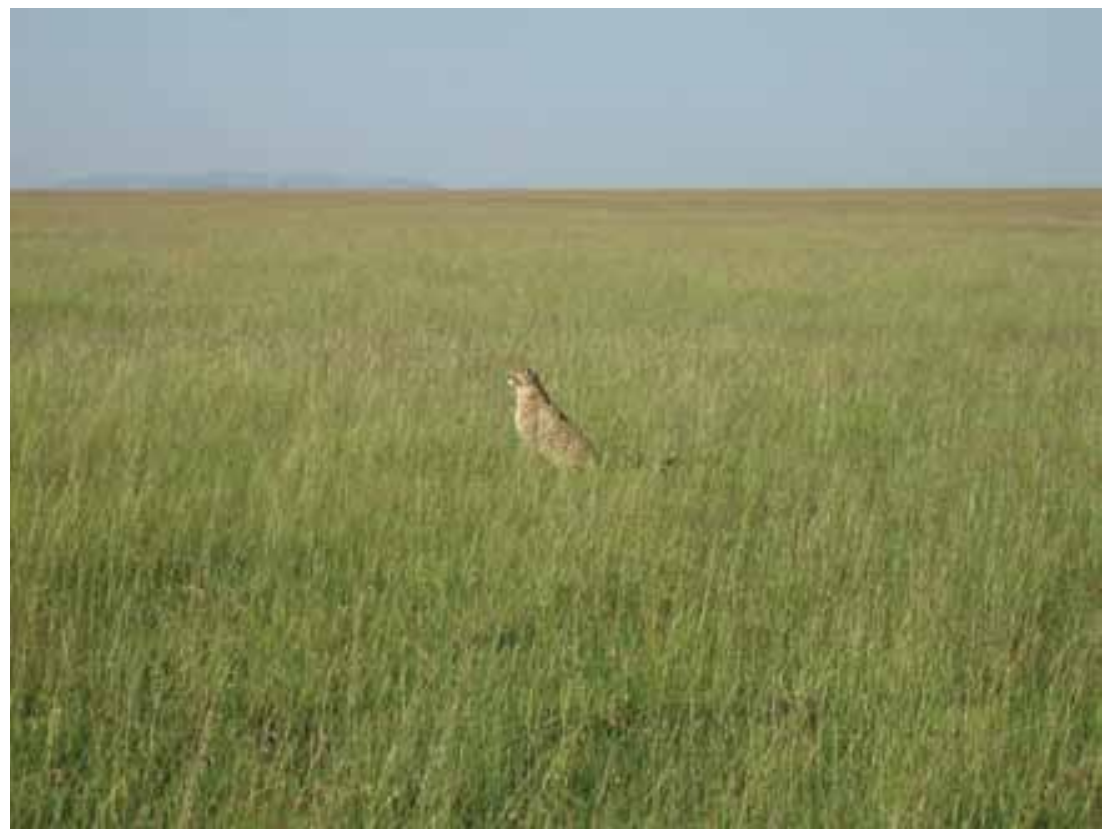

